\title{
CUMULATIVE AUTHOR INDEX (Volume 11)
}

Abdallah, M. S. 427

Abdel-Rahman, M. A. 427

Abouelhassan, S. 83, 199

Adhikari, S. $\quad 553,559,585$

Adhikary, S. 553, 559, 585

Ahonen, M. 457

Arabski, J. 563

Atuchin, V. V. 191

Azizi, A. 563

Badawi, E. A. 427

Badawi, E. 167, 451

Bartoš, I. 447

Belhadi, M. 87, 99, 321

Belykh, S. F. 391

Bhavsar, S. R. 443

Bilić, A. 185

Borca, C. N. 531

Boumrar, H. 155

Bozzolo, G. 235, 591

Brongersma, H. H. 341

Burghaus, U. 521

Canto, G. 77

Canzian, A. 235

Carabineiro, S. A. C. 385

Cattani, M. 223, 283, 463

Ch. Argirusis 419

Chadli, R. 321

Chai, J. C. 217

Chen, G. 15

Chen, Q. 305

Chen, X. 15

Cheng, J. 27

Cheng, X. 211

Chou, W.-C. 229

Creemers, C. 341

Dai, C. Y. 433

Dai, X. Q. 1

Dang, H. 111

Dashti, A. 137

Dashti, M. 33

de Groot, F. M. F. 385

Deng, D. 599

Desai, C. F. 443

Dew, S. K. 391

Dinia, A. 563

Dong, S. 205

Dowben, P. A. 531

Du, W. 379, 491
Duan, C.-G. 531

El-Mansy, M. K. 199

Elmansy, M. 83

Fan, D. B. 27

Farías, D. 591

Farias, R. J. C. 223

Foulias, S. D. 419

Ganesan, V. 265

Gasmi, A. 337

Geng, Y. L. 71, 379, 491

Gesari, S. B. 145

Gunes, M. 49

Güneş, M. 403

Guo, L. X. 367

Guo, L. 291, 497

Gupta, V. K. 41

Han, J. 211, 503

Hannech, E. B. 337

Hardt, D. E. 217

Hasegawa, N. 553

He, Z. 7

Helfensteyn, S. 341

Hirsimäki, M. 457

Huang, S. Y. 515

Hush, N. S. 185

Iqbal, K. 117

Irurzun, I. M. 57

Jeong, H.-K. 531

Jiang, B. L. 367

Jiang, D. 205

Jiang, S. 433

Jimbo, T. 553, 559, 569, 577, 585

Joshi, K. P. 41

Juan, A. 145

Junell, P. 457

Kamaratos, M. 419

Kasapoglu, E. 49, 403, 411

Kato, S. 553

Kesler, V. G. 191

Kh. Zakeri 137

Khan, K. M. 117

Khater, A. 87, 99

Khirman, M. A. 259

King, D. A. 57

Kjeldgaard, L. 385

Komesu, T. 531
Kumar, U. 451

Lacharme, J.-P. 21

Lam, Y. C. 217

Lamoudi, N. 337

Lei, T. Q. 367

Lei, T. 291, 497

Li, D. Y. 173

Li, H. 211

Li, J.-S. 509

Li, Q. 111, 503

Li, T. B. 71

Li, W. 173

Li, Y.-R. 509

Lim, G. C. 305

Lin, J. S. 229

Liu, H. Y. 379, 491

Liu, J. 531

Liu, R. 291, 497

Lu, M.-W. 331

Luyten, J. 341

Mao, L. 111

Maschke, K. 87

Mei, W. N. 531

Meng, H. 599

Miao, Y. 7

Miranda, R. 591

Mishra, R. K. 271, 541, 607

Misra, K. D. 271, 541, 607

Mokutani, H. 553

Mola, E. E. 57

Mosca, H. O. 235

Moshfegh, A. Z. 33

Mukhopadhyay, P. K. 451

Murukeshan, V. M. 217, 259

Ng, V. M. 515

Nieuwenhuys, B. E. 385

Omer, A. M. M. 553, 559, 585

Ozturk, E. 297

Pagura, C. 391

Palmstrøm, C. J. 531

Pan, D. 503

Pan, Y. 433

Pandey, R. P. 607

Park, B. 179, 373

Peng, C. 485, 599

Petukhov, A. G. 531

Pokrovsky, L. D. 191 
Pronsato, M. E. 145

Qin, X. 205

Rafil, O. 155

Rafti, M. 57

Rathore, M. K. 41

Rathore, V. 41

Read, M. N. 447

Reimers, J. R. 185

Romero, M. T. 77

Rubensson, J.-E. 385

Rusop, M. 553, 559, 569, 577, 585

Ryu, Y. 373

Salman, F. 83, 199

Salvadori, M. C. 223, 283, 463

Sari, H. 49, 297, 403, 411

Schultz, B. D. 531

Schurmans, M. 341

Sheha, E. 83, 199

Shrivastava, S. B. 41

Sinha, O. P. 265

Soga, T. 553, 559, 569, 577, 585

Sökmen, I. 49, 297, 403, 411

Song, S. 485, 599

Soni, P. H. 443

Srivastava, P. C. 265

Stepanova, M. 391

Sun, D. L. $\quad 71,379,491$
Takeuchi, N. 77

Tamine, M. 155

Tan, J. L. 259, 305

Tiwari, R. P. 541, 607

Tolstogouzov, A. 391

Tong, S. Y. 1

Tran, D. V. 217

Uchida, H. $\quad 553,559,585$

Umeno, M. $553,559,569,577,585$

Valden, M. 457

Vaz, A. R. 223

Vázquez de Parga, A. L. 591

Vicente, J. L. 57

Vlachos, D. 419

Voronkova, V. I. 191

Wang, B. 15

Wang, G. 15

Wang, H. 27

Wang, J. 503, 521

Wang, X. C. 305

Wang, X. Q. 71, 379, 491

Wang, Y. L. 491

Wang, Y. M. 367

Wang, Z. 211, 503

Wu, H. S. 1

Xie, M. H. 1
$\mathrm{Xu}$, D. $\quad 71,379,491$

$\mathrm{Xu}, \mathrm{M} . \quad 515$

$\mathrm{Xu}$, S. H. 1

$\mathrm{Xu}, \mathrm{S} . \mathrm{Y} . \quad 515$

Yan, H. 27

Yang, C. 211, 503, 509

Yang, H. 211

Yang, J. 363

Yanovskii, V. K. 191

Yesilgül, U. 411

Yin, W.-G. 531

Yong, K. 179, 373

Yoshimura, M. 27

$\mathrm{Yu}, \mathrm{S} .7$

Yu, W.-F. 509

Yu. Maklakova, N. 191

Zaïbi, M.-A. 21

Zhang, G. H. $\quad$ 71, 379, 491

Zhang, S.-M. 311

Zhang, T. 363

Zhang, X. 7

Zhang, Y. C. 27

Zhang, Z. 111

Zhao, J. 15

Zheng, H. Y. 217, 259, 305

Zhou, Y. C. 433 


\section{CUMULATIVE AUTHOR INDEX \\ (Volume 12)}

Abdallah, M. S. $\quad$ 197, 203, 463, 519, 545

Abdel-Latif, R. M. 197

Abdel-Rahman, M. A. 197, 203, 463, 519, 545

Abdulrazaq, O. A. $\quad 299,515$

Adachi, M. 19

Adhikari, S. 13

Adhikary, S. 13

Avilés, F. 101

Azizi, A. 391

Badawi, E. A. 1, 197, 203, 463, 493, 519, 545

Bao, Q. 539, 597, 773, 819

Belash, V. P. 713

Boumrar, H. 85

Cattani, M. 221, 675

Ceh, O. 101, 289

Chai, J. C. 351

Chen, C. 401, 505, 539, 555, 597, $773,781,819$

Chen, C. Z. 41, 123, 161, 209, 369, 443, 741

Chen, G. 279, 417

Chen, J.-X. 753

Chen, L. 539, 597

Chen, L. X. 741

Chen, Y. 709

Chua, S. J. 651

Corona, J. E. 289

Cui, Y.-J. 753

Dai, S. $\quad 59,749$

Dai, X.-Q. 483

Dai, Z. 397

Ding, P. 75

Dinia, A. 391

Dong, Q. 555, 781

Dong, S. M. 549

Drofenik, M. 97, 239

Du, Z. 59

Fang, Z. $\quad 355,489$

Feng, T. 733

Fenineche, N. 391

Filardo Bassalo, J. M. 221

Fort, T. 457

Foulias, S. D. $\quad 721,787$

Gao, L. $\quad 355,489$

Gao, S. 433
Ge, H.-L. 753

Geng, Y. L. 413

Gesari, S. B. 227

Ghodsi, F. E. $\quad 425,793$

Gu, S. 409

Hamzah, E. 631

Han, J. 409

Hashim, A. H. 631

He, P. 749

Huang, S. 397

Huo, Q. H. 41, 161, 209, 443, 741

Irigoyen, B. L. 227

Ismail, R. A. $\quad 299,515$

Ji, Q. $\quad 369,555,781$

Jia, F. 489

Jiang, D. 799

Jiang, D. L. 549

Jiang, X. 59

Jimbo, T. 13, 19, 27, 35, 167, 173, 185, 325, 333, 343, 579, 587, 605, 681, 691, 697

Ju, W.-W. 483

Juan, A. 227

Kamaratos, M. 721

Kan, Y. 561

Kang, H. 355

Kasapoglu, E. 155

Kawano, H. 107

Khan, T. 759

Kinugawa, T. 27

Klimova, I. N. 713

Lam, Y. C. 351

Lan, J. 75

Lei, T. $\quad 539,819$

Lei, T. Q. 41, 123, 161, 209, 443

Li, Q. 733

Li, Y. 59

Lim, G. C. 651

Liu, D. 709

Liu, J. 539

Liu, P. 619

Liu, S. 7, 319, 499, 727

Liu, X. 733

Liu, W. 561, 569, 573, 651

Long, J. 397

Lu, M.-W. 67

Lu, Y. 355
Ma, G. 409

Ma, J. 773

Mafakheri, M. 793

Maldonado, R. D. 289

Maqbool, M. 759, 767

Masumdar, E. U. 703

Mishra, R. K. 47

Misra, A. P. 47

Misra, K. D. 47

Mitrokhin, Yu. S. 713

Mohd Toff, M. R. Hj. 631

Mominuzzaman, S. M. 35

Monteiro, P. J. M. 233

Mubarak, A. 631

Natarajan, B. 645

Novinrooz, A. 793

Ng, F. L. 651

Oliva, A. I. $\quad$ 101, 289

Omer, A. M. M. 13

Park, B. 745

Perdikis, A. 787

Qin, X. H. 549

Rafil, O. 85

Ramakrishnan, V. 645

Ramamurthy, S. 645

Riveros, H. G. 289

Rusop, M. 13, 19, 27, 35, 167, 173, 185, 325, 333, 343, 579, 587, 605, 681, 691, 697

Ryou, J. 233

Sahari, A. 391

Salvadori, M. C. 221,675

Sari, H. 155

Schmerber, G. 391

Sharon, M. 579, 587, 691

Shi, J.-J. 433

Shirata, T. 681

Soga, T. $13,19,27,35,167$, 173, 185, 325, 333, 343, 579, 587, 605, 681, 691, 697

Sökmen, I. 155

Song, S. 457

Sun, Y. 397

Tak, Y. 215

Tamine, M. 85

Tan, S. 75, 799 
Tan, X. 611

Tian, Y. S. 41, 123, 161, 209, 369, 443, 741

Tigrine, R. 85

Tong, S. Y. 483

Tripathi, A. K. 47

Uchida, H. 13

Uma, K. 605, 697

Umeno, M. 13, 35, 579, 587, 691

Uskoković, V. 97, 239

Vasu, V. 645

Vlachos, D. $\quad 721,787$

Wang, D. 59, 401, 505, 539, 555, 773, 781, 819

Wang, D. Y. 41, 123, 161, 209, 369, 443

Wang, X. 733

Wang, X. C. 651

Wang, X. Q. 413

Wang, Z. 409

Wu, L.-N. 753

Wu, X. 489
Xiao, B. L. 549

Xiao, H. Y. 809

Xie, M. H. 483

$\mathrm{Xu}$, D. 413

$\mathrm{Xu}$, J. $\quad 561,569,573,733$

Xu, L. 809

$\mathrm{Xu}$, S. 397

Xu, Z. 569

Yahya, K. Z. 299, 515

Yakovkin, I. N. 449

Yang, C. 409

Yang, D. 355, 409

Yang, H. 355

Yang, J. 355, 749

Yang, Y. 409

Yao, L. 773

Ye, Q.-L. 753

Yi, D.-Q. 499

Yi, Y. 799

Yin, M. 355, 489

Yong, K. 215, 745

Yu, G. W. 413

Yu, S.-J. 753

Yu, Z.-M. 499
Zhang, G. H. 413

Zhang, J. 733

Zhang, J. Q. 727

Zhang, L. 433, 819

Zhang, M. 749

Zhang, N. 709

Zhang, S. 115, 145, 359, 379, 523, 623

Zhang, T. 355,489

Zhang, W. 7

Zhang, X. 59

Zhang, X. P. 279, 417

Zhang, Y. 457

Zhang, Z. 749

Zhao, Y. 401, 505, 597

Zheng, H. Y. 351

Zheng, J. 355, 489

Zheng, X. J. 611

Zhou, Y. C. 611

Zhou, Z. 75, 799

Zhu, H. 351

Zou, S. 733

Zu, X. T. 809 


\section{CUMULATIVE AUTHOR INDEX \\ (Volume 13)}

Abdel-Rahman, M. A. 485

Abdelhamed, M. O. 485

Abdullah, S. 1, 7, 573, 577, 585,593

Abo-Elsoud, M. 773

Adhikari, S. $\quad$ 577, 585, 593, 789

Adhikary, S. 789

Ali, M. 763

Anwar, M. 457

Ashour, A. 69,87

Badawi, E. $\quad 69,485$

Bao, Q. 93, 103, 451, 655

Bao, R. 509, 645

Bastrukov, S. 81

Borstel, G. 149

Brack, N. 319

Braun, W. 155

Brizuela, G. 387

Canto, G. 495

Cao, N. 423

Chen, C. 35, 63, 93, 103, 451, 503, 509, 545, 551, 645, 655, 687,737

Chen, G. 613

Chen, J.-X. 779

Chen, L. B. 535

Chen, L. $\quad 545,551$

Chen, S. 669

Chen, T. 123

Chen, Y.-M. 423

Chen, Y. W. 123, 833

Chen, Z. Y. 57

Cheng, M. F. 519

Cheng, Y.-Y. 265

Cheng, Z. 313

Chihaya, H. 201

Chung, T.-W. 279

Cortie, M. B. 297

Dai, C.-A. 265

Dai, X. 429

Daimon, H. $\quad$ 191, 197, 209

Damiani, D. 387

Dawson, P. 273

Ding, E. 819

Dong, Q. 35, 63, 503, 509

Dong, Z. C. 143

Edamoto, K. 185, 227

Eftekhari, A. 703, 753, 809
Eglitis, R. I. 149

El-Sayed, N. Z. 69

Feng, P. X. 711, 847

Ford, M. J. 297

Fu, P. 429

Gard, F. S. 215

Ge, H.-L. 779

Geng, Y. L. 111, 607

Ghauri, I. M. 457

Gong, J. 369

$\mathrm{Gu}$, S. 13

Guo, F. Z. 209

Guo, P. S. 123

Guo, W. F. 111

Guo, X. L. 143

Guo, X. P. 57

Hamzah, E. 413, 621, 763

Han, J. 13

Hashem, H. A. 439

Hattori, K. 191, 209

He, P. 51

Hirose, K. 179, 309

Honda, M. 197

Hou, J. G. 143

Hou, X. Q. 111

Huang, S. M. 833

Huang, S. Y. 833

Huang, S. 123

Huang, W. 251

Ishikawa, H. 329

Ismail, H. $\quad 773$

Jayavel, R. 803

Ji, Q. 35

Jia, Y. 841

Jian, X. G. 733

Jiang, F. 13

Jiang, Z. 471

Jimbo, T. 1, 7, 577, 585, 593

Jin, C. X. 833

Juan, A. 387

Kamaratos, M. 681

Kamiko, M. 201

Kang, E.-T. 251

Kang, E. T. 313

Kanno, I. 167

Kasapoglu, E. 397, 747
Kashyap, S. 221

Kataoka, K. 191, 209

Kato, M. 185, 227

Kato, Y. 209

Kawaguchi, T. 235

Kazemzad, M. 703, 753

Kera, S. 259

Keyanpour-Rad, M. 703, 753

Kim, J. 567

Kobayashi, E. 259

Kobayashi, N. 179, 309

Kojima, I. 201

Kou, Y. 733

Kushvaha, S. S. $\quad 241,287$

Lai, P.-Y. 81

Lee, K. Y. 635

Lei, T. $\quad 451,655$

Lei, T.-Q. $\quad 545,551$

Li, D. J. 173

Li, H. Y. $\quad 847$

Li, J. 841

Li, M.-S. 423

Li, W. 819

Li, X. D. 123, 833

Li, Y. 613

Li, Y. R. 27

Li, Z. 403

Lian, J. 471

Liesegang, J. 319

Lim, D. 795

Liu, J. $\quad 451,841$

Liu, P. 785

Liu, Q. X. 173

Liu, S. 661

Liu, W. 403

Liu, X. 471

Liu, X. J. 607

Liu, Y. 815

Liu, Z. Y. 733

Long, J. 123

Lu, M. 599

Lü, Y.-P. 423

Luan, Y. 429

Luo, X. D. 519

Ma, G. 13

Ma, J. 93, 103, 687

Ma, Q.-S. 423

Ma, X. G. 825

Maldonado, R. D. $\quad 557$

Markovic, N. M. 697 
Mase, K. 259

Masens, C. 297

Matsui, F. 197, 209

Matsukawa, H. 235

Matsushita, T. 209

Mori, E. E. 681

Mubarak, A. 413, 621

Muhamad, M. R. 573

Mun, B. S. 697

Murthy, L. C. S. 357

Murugakoothan, P. 803

Nakano, S. 329

Natarajan, B. 351

Neoh, K. G. $\quad 251,313$

Ni, C. 123

Nie, J. L. 45

Nishimura, T. 191

Noh, D. Y. 167

Ogiso, H. 329

Oh, S. 201

Okudaira, K. K. 259

Oliva, A. I. 557

Omer, A. M. M. 577, 585, 593

Ordejón, P. 495

Osovskii, V. D. 375

Ozaki, K. 329

Ozaki, T. 309

Ozawa, K. 185, 227

Park, Y. W. 635

Pei, Z. 369

Peng, J.-W. 251

Petrova, N. V. 375

Pigram, P. J. 319

Ploog, K. H. 155

Podder, J. 1, 7

Prince, K. 215

Prissanaroon, W. 319

Ptushinskii, Yu. G. 375

Qi, B. 103, 545, 645

Que, W. X. 123

Ramakrishnan, V. 351

Ramamurthy, S. 351

Rao, K. N. 221, 357

Rathnakumari, M. 803

Refat, M. S. 439

Riley, J. D. 215

Riveros, H. G. 557

Ross, Jr., P. N. 697

Rossi, M. 697

Rusop, M. 1, 7, 573, 577, 585, 593

Ryou, J. 345
Sankar, R. 803

Sankara Narayanan, T. S. N. 635

Santoso, E. G. 795

Sari, H. 397, 747

Sato, T. 185,227

Sellai, A. 273

Seo, S. H. 167

Shah, S. 345

Shamala, K. S. 357

Shi, H. 149

Shi, Y. C. 847

Shi, Z. L. 313

Shimamoto, Y. 191

Siddheswaran, R. 803

Siddiqi, S. A. 457

Simonetti, S. 387

Sobhy, M. S. $\quad 479,773$

Soga, T. 1, 7, 577, 585, 593

Sökmen, I. 397, 747

Song, M. 687

Song, X. 117

Song, Y. 841

Song, Y. Y. 607

Stamenkovic, V. 697

Sugimoto, W. 201

Sui, J.-L. 423

Sun, C. 369

Sun, Z. 123,833

Sureshkumar, P. 803

Suzuki, T. 167

Tang, C.-Q. 825

Teh, K. M. 795

Tian, Y. 551

Toff, M. R. M. 413, 621, 763

Tsui, T.-A. 265

Uchida, H. 789

Ueno, N. 259

Umeno, M. 577, 585, 593, 789

Vasu, V. 351

Wakayama, Y. 143

Wan, S. 795

Wang, B. 117

Wang, D. 35, 63, 103, 451, 503, $655,669,687,737$

Wang, H. 369, 737

Wang, J. 815

Wang, J. Y. 733

Wang, L. L. 123

Wang, M. X. 173

Wang, Q. 329

Wang, Q.-X. 423

Wang, S. 841
Wang, X. 503, 613, 841

Wang, X. Q. 111, 607

Wang, X.-S. $\quad 241,287$

Wang, X. Z. 123

Wang, Z. 759

Wang, Z. G. $\quad 45,365$

Wang, Z. Y. 607

Wasa, K. 167

Watanabe, M. 697

Wu, H. 279

Wu, H. S. 815

Xiao, H. Y. 45

Xiao, W. 241, 287

Xie, M. H. 815

$\mathrm{Xu}$, D. 111,607

$\mathrm{Xu}, \mathrm{H} . \quad 13,369$

$\mathrm{Xu}$, J. 201

Xu, M. 287

Xu, M.-J. 241

$\mathrm{Xu}, \mathrm{S} . \quad 123$

Xue, S. 759

Yakovkin, I. N. 375

Yamamoto, R. 201

Yan, H. 117, 369

Yan, Q. L. 733

Yan, Z. 241, 287

Yang, B. Q. 847

Yang, C. 13, 27

Yang, D. 13, 51

Yang, J. 51

Yang, J. H. 519

Yang, M. 403

Yang, X.-H. 825

Yao, L. 93, 103

Yao, S. 489

Yao, Y. 489

Yesilgul, U. 747

Yi, Y. 27

Yong, K. 567

Yu, G. W. 607

Yu, H. 509, 645

Yu, M. 117, 369

Yu, S. 669

Yu, S.-J. 779

Zhang, G. H. 111

Zhang, H. 471

Zhang, H. B. $\quad 111,607$

Zhang, J. J. 173

Zhang, J. Q. 661

Zhang, L. 75, 489, 545, 551, 645,655

Zhang, M. 51

Zhang, Q. 57 
Zhang, S. $\quad 17,127$

Zhang, T. H. 519

Zhang, X. 613

Zhang, Y.-J. 779

Zhang, Z. J. 123
Zhao, X. 841

Zhao, Y. 451, 687

Zheng, H. Y. 795

Zhong, M. 403

Zhou, Y. 117
Zhu, H. B. 833

Zhu, X. 313

Zu, X. T. $\quad 45,365$ 


\section{CUMULATIVE AUTHOR INDEX \\ (Volume 14)}

Abdel-Hamed, M. O. 43

Anwar, M. 5

Atuchin, V. V. 403

Bao, R. 49

Belkaid, M. S. 165

Benreguia, N. 165

Bi, J. Q. 17

Biskupski, P. 357

Bo, W. 371

Bouhafs, D. 165

Brizuela, G. 209

Brown, I. G. $\quad 87,345$

Cao, N. 371

Cattani, M. 87, 345

Chen, C. $\quad 49,283$

Chen, C. Z. 361

Chen, C.-Z. 179

Chen, J. 111

Chen, L. 241

Chen, Y. 23, 241, 411

Chen, Y. W. 135, 261

Cheng, G. A. 193

Cheng, M. F. 517

Cheng, Q. J. 225

Cheng, R. M. 135

Chu, J. B. 225

Cottam, M. G. 471

Czajka, R. 357

Damiani, D. 209

Dang, X. 49

Das, T. K. 471

Deng, Q. 23, 241

Dennis, G. R. 235

Ding, H. F. 71

Ding, Y. 123, 277, 435

Dong, Y. 171

Dowben, P. A. 481

Du, B. 315

Dwivedi, A. 57

El-Tayeb, M. S. N. 489

El-Tayeb, N. S. M. 185

Fan, H. L. 439

Fan, R. H. 17

Fan, S. 123, 277, 435

Fang, K. 111

Feng, P. X. 309
Ghauri, I. M. 5

Ghodsi, F. E. 219

Gonzalez, E. A. 79

Hai, Z. 371

Hamzah, E. 93

Han, J. 147

He, F. 71

He, J. 171,301

He, X. 241

He, Y. 499

Hu, G. $\quad 123,277,435$

$\mathrm{Hu}$, J. $\quad 129,301$

Huang, S. M. 135, 261

Huang, S. Y. 225

Huang, Y. 71

Jasen, P. V. 79

Jeyakumaran, N. 293

Jiang, S. W. 141

Jimbo, T. 425

Jin, C. X. 261

Juan, A. 79, 209

Kannangara, G. S. K. 235

Katircioğlu, Ş. 507

Kesler, V. G. 403

Khayatiyan, S. A. 219

King, B. V. 31

Kong, Y.-H. 255

Li, C.-S. 255

Li, D. 101

Li, F. 499

Li, H. 129

Li, J. $\quad 117,199$

Li, J.-G. 445

Li, M. G. 135

Li, M.-S. 371

Li, Q. 315

Li, X. D. 261

Li, X. J. 193

Li, Y. R. 141

Liang, H. 71

Liao, S. 411

Liu, A. D. 193

Liu, J. 283

Liu, P. 269

Liu, X. J. 431, 439

Liu, Z. 329

Long, J. D. 225
Lu, Y.-P. 179

Luna, C. R. 79

Luo, X. D. 517

Ma, J. 49

Mackenzie, K. 229

Mahiou, L. 165

Miao, Y. 111

Mielcarek, S. 357

Milev, A. S. 235

Moussi, A. 165

Mubarak, A. 93

Naksata, M. 229

Nasiruddin Khan, M. 461

Natarajan, B. 293

O'Connor, D. J. 31

Ouyang, Z.-Q. 129

Pan, L. K. 135

Pang, L. X. 17

Parasyuk, O. V. 403

Pi, T.-W. 377

Ramamurthy, S. 293

Ren, S. 17

Rusop, M. 425

Salvadori, M. C. $\quad 87,345$

Samavat, F. 31

Sarwar, A. 461

Sha, L. 451

Shi, J. Z. 361

Shuttleworth, I. 1

Shuttleworth, I. G. $\quad 321,387$

Siddiqi, S. A. 5

Simonetti, S. 209

Soga, T. 425

Song, J. 117, 199

Song, J.-G. 445

Song, J.-R. 445

Song, M. 283

Song, S. 395

Sui, J.-L. 371

Sun, J. 301

Sun, K. N. 17

Sun, R.-X. 179

Sun, W. 23, 241

Sun, Z. 135, 261 
Sun, Z. H. 439

Szuba, S. 357

Tang, W.-H. 255

Tang, Y. 301

Teixeira, F. S. $\quad 87,345$

Thountom, S. 229

Toff, M. R. M. 93

Tran, N. H. 235

Tunkasiri, T. 229

Uma, K. 425

Vasu, V. 293

Vaz, A. R. 87

Wan, Y. Z. 71

Wang, A.-J. 179

Wang, D. 49

Wang, F. 329

Wang, P. 123, 435

Wang, T. 269

Wang, X. Q. 431, 439

Wang, X. Z. 135

Wang, X. 315
Wang, Z. Y. 431

Wiederkehr, R. S. $\quad 87,345$

Wilson, M. A. 235

Winiarz, S. 357

$\mathrm{Wu}, \mathrm{H} .123$

Wu, W. 123

$\mathrm{Wu}, \mathrm{X} .111$

Wu, X. L. 193

Wu, Y. 361

Xiao, L. 171

$\mathrm{Xu}, \mathrm{D} . \quad 431,439$

$\mathrm{Xu}$, J. 329

$\mathrm{Xu}, \mathrm{S} . \quad 225$

Xu, X. B. 225

Xue, W. B. 193

Yakovkin, I. N. 481

Yan, D. 171

Yang, B. Q. 309

Yang, C. 147

Yang, D. 147

Yang, J. H. 517

Yao, L. 49

Yao, L. G. 261
Yao, M. 499

Yin, X. 147

Yousif, B. F. 185, 489

Yu, G. W. 431, 439

Yu, G. 439

Yu, T. C. 377

Yuan, C. 147

Zhang, F. 301

Zhang, G. H. 431, 439

Zhang, G.-L. 255

Zhang, L. 117, 151, 199

Zhang, L.-M. 445

Zhang, Q. Y. 141

Zhang, S. 361

Zhang, T. H. 517

Zhang, X. 193, 301

Zhang, X.-D. 129

Zhang, Y. 129, 283, 395, 499

Zhdanov, V. P. 419

Zhu, L. Y. 431

Zhu, L. 171

Zhu, Y. L. 439

Zou, Z. 315 